\title{
Le pouvoir disciplinaire chez Calvin
}

\author{
MARTA GARCÍA-ALONSO
}

UNED-Filosofía Moral y Política

This paper discusses the doctrinal foundations of criminal law in Calvin's ecclesiology, namely his theology of the original sin, and its practical implementation in Geneva's consistory. On these grounds, I analyse the distinction between civil and ecclesiastical criminal law. Both State and Church were granted by Calvin a ius gladii, but only the former can claim a right to impose physical punishment, whereas the latter should just punish spiritually. Here lays, in my view, the difference between law and discipline.

\section{Le pouvoir disciplinaire chez Calvin est-il un vrai droit?}

T'examen protestant est généralement compris comme la liberté individuelle Lreconnue au chrétien de lire et d'interpréter la Bible. Or, cette conception de l'examen, à laquelle on est si habitué aujourd' hui, fut à lorigine celle des polémistes catholiques, qui firent promptement réagir les premiers réformateurs ${ }^{1}$. Il s'agit néanmoins de la lecture la plus répandue chez les philosophes politiques et du droit, pour qui l'examen protestant est à lorigine de la liberté de conscience soutenue autant par bon nombre de théories constitutionnelles modernes que par certaines déclarations des droits.

Certes, dans la mesure où le protestantisme avait miné le monopole du Magistère Catholique sur l'interprétation de l'Écriture, il renferme une semence de modernité. On oublie cependant que, historiquement, le problème soulevé par les premiers réformateurs était celui de juger si la conduite du fidèle était conforme ou non à la Bible. Or, la conjonction du principe de la sola fides et de celui de la liberté du chrétien vis-à-vis du droit posait une question importante : comment faire pour combler le hiatus ainsi créé entre la conscience et laction? Il ne faut pas oublier que le sens originaire de léthique chrétienne était l'exigence d'une adaptation interne de l'individu à la volonté divine. 
Pourtant, la conformité de l'individu à la norme ne peut être jugée que par Dieu, comme c'est le cas dans le cadre protestant. L'action ne pourra être supervisée et contrôlée que si la loi morale a une dimension publique. Mais comment et conformément à quoi peut-on juger des actes humains ? Par quel moyen peut-on contrôler si la conduite des croyants est conforme aux préceptes bibliques sans aller contre leur intimité religieuse, contre leur conscience? Une fois niés la confession auriculaire et le sacrement de la pénitence, l'examen ne pouvait pas s'en remettre tout simplement à la conformité personnelle du croyant au texte sacré ; l'examen supposait une dimension supra individuelle. Laquelle?

Forrester maintient que là où Calvin se sert de la loi morale pour doter l'Écriture d'un contenu positif - en y voyant un modèle d'action —, Luther n'y trouve qu'une simple fonction régulatrice ${ }^{2}$. Nous ajouterons pour notre part que cette affirmation ne prend son plein sens que si on accepte que ce modèle d'action exige que les préceptes moraux bibliques aient une forme publique. L'établissement de lois qui soient en accord avec les principes chrétiens devient impératif à partir du moment où l'on défend l'idée que, non seulement la conscience du fidèle, mais aussi sa conduite extérieure doivent se conformer à la volonté divine ${ }^{3}$. Cependant, cette forme publique relève-t-elle strictement du droit?

Dans cet article nous allons voir la façon dont la conscience réformée s'est dotée d'un support disciplinaire pénal qui fut considéré comme une part intégrante de la conception calvinienne de la vie communautaire. Nous constaterons que tout cela s'est fondé sur une formulation légale des principes moraux, qui soutient l'interprétation du troisième usage de la loi morale (didactique) comme modèle d'action pour le chrétien. De même, nous allons tenter de montrer de quelle façon l'éthique protestante est solidaire d'une juridiction d'abord ecclésiastique et disciplinaire, mais aussi étatique, cette dernière étant conçue comme droit dans un sens strict.

\section{Le troisième usage de la loi morale : les fondements ${ }^{4}$}

D'après la théologie de Calvin, la loi divine renferme la législation biblique comme un tout, c'est-à-dire comme la somme des préceptes cérémoniels, judiciaires et moraux accordés au peuple juif en tant que Loi nouvelle ${ }^{5}$. 
Néanmoins, en accord sur ce point avec la tradition catholique, le réformateur français ne reconnaît pas le même statut à toutes les lois de l'Ancien Testament. Avec l'établissement du Nouveau Testament, l'usage de deux des formes de la loi fut abrogé puisqu'elles furent données aux juifs en tant que lois particulières : les lois cérémonielles et judiciaires ont cessé dêtre appliquées, selon Calvin, sans que ni la religion ni la politique ne s'en ressentent. Malgré tout, ces cérémonies et ces lois politiques gardent dans leur contenu quelque degré de vérité, qui peut être retrouvé au travers d'une interprétation adéquate, et ce, même si elles ont perdu la forme dans laquelle elles furent instituées chez le peuple juif ${ }^{6}$.

Cependant, il y a une loi qui reste en vigueur : la loi morale. La validité de cette loi, pourtant, ne veut pas dire que n'importe qui peut être sauvé en la suivant. Cela dit, le fait que cette loi ait perdu sons sens sotériologique n’implique pas qu'elle n'ait pas encore un rôle important à jouer, puisque, conformément à la doctrine morale calvinienne, la loi morale est le seul modèle de justice qui doit guider le chrétien en tout moment et en tout lieu. Certes, au début, la connaissance directe de cette loi était possible dès lors qu'elle était imprimée « au cœur de tous les hommes » mais, après la chute, la Bible devient le seul moyen d'y accéder ${ }^{7}$.

La loi morale peut se subdiviser selon ses différents sens ; des sens qui, par ailleurs, sont en correspondance avec ses fonctions : délation, politique et pédagogique $^{8}$. La fonction théologique ou la fonction de délation ${ }^{9}$ a pour but de montrer aux chrétiens leurs péchés, ainsi que leur impuissance à accomplir par eux-mêmes la volonté divine ${ }^{10}$. La fonction politique a pour objectif de favoriser un minimum d'ordre social : en faisant partie des structures du siècle, on s'expose (en effet) aux conséquences du péché et du désordre ; la fonction politique de la loi n'a d'autre but que de freiner les effets de ces derniers ${ }^{11}$. La troisième fonction de la loi morale, ou fonction pédagogique, constitue le fondement d'une éthique chrétienne définie comme la seule règle valable en tout temps et lieu, et à laquelle le fidèle est obligé de se plier:

Et de fait, si nul ne peut nier qu'en la Loy il n'y ait comme une image entière de parfaite iustice, ou il faudra dire que nous ne devons avoir nulle reigle de bien vivre, ou qu'il nous faut tenir à icelle. Car il n'y a point plusieurs reigles de bien vivre, mais une seule, qui este perpétuelle et immuable. Pourtant? ce que dit David, que l'homme 
iuste médite iour et nuit en la Loy (Sal.1,2), ne doit estre rapporté à un siècle, mais convient à tous aages iusques en la fin du monde. ${ }^{12}$ Une fois établis les objectifs, il faut interpréter la loi morale en corrélation avec les actions auxquelles elle veut mener : des actes extérieurs - sur le plan de la loi humaine - , et des actes intérieurs pour lesquels la loi fait appel à la conscience et exige que l'on réfrène les intentions et les pensées contraires à la volonté divine ${ }^{13}$. Plus encore : d'après Calvin, il faut dépasser le sens littéral de la loi afin de comprendre ce qu'elle exige dans toute son étendue : ses préceptes commandent non seulement ce qui est explicitement dit, mais interdisent le contraire, et vice-versa. Par conséquent, il faut interpréter les règles morales comme des synecdoques, c'est-à-dire qu'on doit prendre la partie pour le tout, car la Loi morale ne requiert pas seulement l'abstention, mais exige une conduite vertueuse. On doit aussi savoir que la loi morale suppose larticulation des deux Tables, lesquelles résument les devoirs éthiques et religieux du chrétien d'une manière exemplaire : la première Table commande le devoir d'adorer Dieu avec piété (pietas) - religio —, et la deuxième fait appel à la charité - aequitas -, car elle ordonne d'aimer son prochain avec sincérité. Enfin, le plus important est que la deuxième Table doit toujours trouver son sens dans la Première et sétayer sur elle ${ }^{14}$.

De fait, le sens originel de l'éthique chrétienne suppose une adéquation intérieure de l'individu à la volonté divine; cependant elle exige aussi de lui une adéquation extérieure, au niveau de sa conduite. Mais il faut remarquer que, dans le cadre du protestantisme, où l'accommodement intentionnel de l'individu ne peut être jugé que par Dieu, l'action du chrétien ne peut être supervisée et contrôlée que si on transpose la loi morale dans une dimension publique $^{15}$. De quelle façon et conformément à quoi peut-on juger des actions humaines ? Par quel moyen peut-on savoir avec certitude si la conduite des croyants est conforme aux préceptes bibliques, et ce sans attenter à leur intimité religieuse ? À notre avis, Calvin fut conscient de ce problème, puisque, de même que saint Augustin ${ }^{16}$, il reconnut que la loi se réfère de manière directe à la conduite du fidèle, et seulement de façon indirecte à leur intention. Plus explicitement, saint Thomas souligna que la différence entre le devoir moral et le devoir légal résidait dans la démarcation entre ce que le juge peut juger et ce qui ne peut être jugé que par Dieu ${ }^{17}$. De la même façon, pour le réformateur français, on ne peut juger des autres individus quà partir de leurs actions ${ }^{18}$, puisque les lois humaines ne contraignent pas la conscience mais visent à main- 
tenir le bon ordre parmi les individus. Cela est vrai pour les lois politiques, mais aussi pour les lois ecclésiastiques.

À partir du moment où l'on défend l'idée que ce n'est pas la seule conscience du fidèle qui doit se conformer à la volonté divine, mais aussi sa conduite extérieure - à laquelle renvoie l'action —, se fait jour la nécessité de disposer de lois accordées aux principes chrétiens. Et c'est précisément le droit qui permet aux principes éthiques individuels d'acquérir un caractère public à partir duquel on pourrait exercer un contrôle institutionnel. De quelle autre façon, sinon, pourrait-on vérifier que la loi morale est bien, comme l'exige Calvin, le guide de la vie du chrétien ? De sorte que les lois humaines, pour bien être chrétiennes, doivent se conformer à la Volonté de Dieu. Et c'est là précisément la troisième fonction de la loi morale : offrir le fondement théologique nécessaire à ce droit proprement chrétien. Soulignons la nuance : Calvin défend un droit fondé dans la Bible, inspiré par elle, mais non identifié avec elle ${ }^{19}$. Pour la plupart des théologiens chrétiens de l'époque, la Bible constituait un code moral et non pas un code légal.

\section{Forme ecclésiologique des préceptes de la loi morale}

Donc, la possibilité, admise par Calvin, d'une loi positive inspirée par l'Évangile a pour fondement doctrinal la présence d'un usage pédagogique de la loi dans sa théologie. Cette troisième fonction détermine la loi morale comme le modèle d'une justice parfaite et le guide de conduite du chrétien. D’autre part, pour ce qui touche à la nécessité des lois dans l'Église, Calvin décrit ainsi la conditio sine qua non de toute constitution ecclésiale légitime :

[I]e n'entend point d'approuver autres constitutions que celles qui sont fondées en l'authorité de Dieu et tirées de l'Escriture, tellement qu'on les puisse totallement appeller Divines. [...][La costume de nous agenouiller quend on fait les prières solennelles] Ie dy qu'elle este tellement humaine qu'elle est aussi divine. Elle est de Dieu, entant qu'elle est partie de ceste honnêteté laquelle l'Apostre nous recommande (1 Co., 14, 40). Elle est des homme, entant qu'elle nous monstre spécialement et par exprès ce qui avoit seulement esté touché en général par l'Apostre. [...] Quant à la discipline externe 
et aux cérémonies, il ne nous a point voulu ordonner en particulier et comme de mot à mot comment il nous faut gouverner, d'autant que cela dépendoit de la diversité des temps, et qu'ne merme forme n'eust pas esté propre ny utile à tous aages. Donc il nous faut avoir recours à ces reiles générales que i’ay dit : c'est assavoir que tout se face honnestement et par ordre en l'Église. ${ }^{20}$

Dans l'édition de l'Institution de 1539 se trouve reconnue la nécessité d'une constitution spécifique à l'Église, étant donné que nul ordre, y compris lordre ecclésial, ne peut être établi sans lois. L'édition de l'Institution de 1545 incorporait une grande nouveauté : ces lois-là surgissent, selon Calvin, grâce au pouvoir législatif de l'Église ${ }^{21}$. Ce serait celle-ci - et non pas l'autorité civile qui aurait la responsabilité d'instaurer des lois susceptibles de promouvoir l'honnêteté et la concorde au sein de la communauté des fidèles. Il y a deux types de lois ecclésiastiques : les lois dites cérémoniales, qui régulent les rituels et la forme dans laquelle les sacrements doivent être administrés, et les lois disciplinaires, qui dictent la conduite des ministres et des fidèles conformément à la Loi de Dieu. Le pouvoir législatif ecclésial s'occupe de l'adaptation de la loi divine à des temps et à des lieux concrets, et de sa traduction juridique en matière cérémonielle et disciplinaire dans un contexte et un temps déterminés.

D'autre part, les lois ecclésiales font exclusivement appel à l'obéissance extérieure du chrétien, vu qu'elles ont sa source dans le législateur humain, lequel détermine en particulier ce que l'Écriture établit en général. De même, il s’agit de lois qui ne peuvent pas toucher le for interne du croyant mais seulement son for externe, puisque Dieu seul exerce une justice sur les consciences. Contrairement aux lois catholiques ${ }^{22}$, nulle loi ecclésiale humaine ne peut prétendre à la vie éternelle.

Certes, la création d'un corps ecclésiastique auquel on devait reconnaitre le droit de légiférer sur sa propre communauté religieuse, était une revendication qui dépendait en dernier ressort d'un pouvoir exécutif civil, toujours disposé à réviser ces prétentions. Car si c'était théoriquement à l'Église que devait aller le pouvoir législatif, la promulgation des lois dépendait, en dernier ressort, du pouvoir civil - ce fut le cas des Ordonnances ecclésiastiques rédigées par Calvin lui-même. Toutefois, il ne s'agissait pas d'un problème spécifique de l'Église calvinienne, mais de toute organisation prétendant s'autoréguler dans 
le cadre de l'État dès le XVI e siècle. De toute façon, la reconnaissance par Calvin d'un pouvoir législatif propre à l'Église suffit à notre argumentation.

Mais l'Église peut-elle aussi juger de la transgression de ces lois ? Et si on l'admet, quel est le fondement de ce pouvoir?

\section{Le fondement du droit pénal ecclésial}

Selon Prodi, c'est Melanchthon qui aurait été le plus réceptif à la diffusion par la papauté du XVe siècle (et en particulier par Pie II) d'une figure moderne du prince, vu comme un pasteur responsable de la vie temporelle et spirituelle de ses sujets, comme le gouverneur d'un Tempelstaat où il n'y aurait pas de séparation entre le bien temporel et spirituel des sujets. Ce pasteur gouvernerait donc non seulement au travers de préceptes (praeceptio) mais aussi au moyen de l'enseignement et de la discipline (instructio) ${ }^{23}$.

On pourrait dire que, dans la défense du pouvoir pénal, l'Église protestante suivit une argumentation semblable à celle de la tradition catholique. Raphaël Eckart maintient que l'origine du débat se trouve dans l'émergence du droit pénal canonique comme champ différencié du péché et dans le nouveau rôle pénitentiel qu'assume l'Église à partir du XII ${ }^{e}$ siècle ${ }^{24}$. La pénitence permet de réduire ou d'éviter la peine que Dieu aurait à infliger car elle a, en tant que sacrement, un effet direct sur le jugement du péché et le salut. D’autre part, le droit réfrène les actions contraires à l'intérêt public, au sens large, et sa fonction sera donc de «maintenir la société et les institutions qui la gouvernent et non d’assurer le salut du délinquant, qui relève de la pénitence $»^{25}$.

Interest rei publice, ne crimina remaneant impunita ${ }^{26}$. Ce qu'il faut purger, c'est l'atteinte contre l'Église, et non pas contre Dieu. Il ne faut pas oublier que le criminel n'a pas seulement commis un péché, mais a perpétré un crime ; et c'est celui-ci qui doit être jugé, car, bien que tout péché ne soit pas considéré comme un crime, tout crime est en revanche un péché. Cependant, si, pour les canonistes, la peine est appliquée en raison du tort causé à l'Église, plus que pour punir la violation d'un commandement divin, aux yeux de Calvin, la fonction de la peine est double : c'est la violation du commandement qui frappe directement l'Église instituée, vu que ses lois sont directement inspirées par l'Écriture ; pourtant, le châtiment prévu pour le crime n'est qu'un châtiment indirect puisque le juste châtiment ne peut être imposé que par Dieu. 
Les effets de la loi doivent donc être tant répressifs qu'exemplaires, mais jamais rétributifs, car, lorsque il s'agit de Dieu, il n’y a pas de proportionnalité entre le châtiment et la violation du commandement.

Certes, les protestants vont nier le sens de la pénitence, et donc toute action institutionnelle sur le for interne; pourtant, en ce qui touche le for externe, Calvin va se battre pour que l'Église obtienne le pouvoir d'imposer ses propres sanctions à l'encontre de tout fidèle qui, par ses actions, contredit ou transgresse les préceptes divins qu'elle a pour charge d'enseigner. De même, il soutiendra l'idée que c'est l'Église elle-même qui doit juger ses ministres lorsqu'ils désobéissent aux normes ecclésiales légitimement fixées par les Ordonnances ecclésiastiques de 1541. Comme nous le rappelle Prodi, on peut faire remonter ces demandes reformées au droit canon antérieur, là où celui-ci était exprimé par le fonctionnement d'institutions ecclésiastiques telles que la paroisse, les synodes, les tribunaux des évêques, etc. ${ }^{27}$

\section{Le pouvoir judiciaire ecclésial et ses institutions}

Nulla poena sine lege ou, selon les mots déjà prononcés par Pierre Lombard au XII ${ }^{e}$ siècle : il n'y a pas de crime sans interdiction préalable ${ }^{28}$. Autrement dit, tout ce qui est considéré comme significatif du point de vue moral doit être sanctionné par la loi pour pouvoir être jugé. Et, pour Calvin, ce que l'on considère significatif sur le plan moral a déjà été sanctionné par la Bible. Le pouvoir législatif de l'Église lui permet de donner une forme actuelle aux matériaux cérémoniels et disciplinaires dont elle a besoin. En plus du pouvoir législatif, Calvin reconnaît à l'Église le pouvoir judiciaire ou disciplinaire qui lui permettra de juger les délits contre les lois ecclésiales établies, ou contre les lois dérivant de n’importe lesquels des préceptes des Deux Tables.

Un chapitre complet de l'Institution est dédié au pouvoir judiciaire ecclésial, ou discipline ${ }^{29}$. La discipline n’y est plus décrite comme les nerfs qui permettent et structurent l'union des membres de la communauté ; autrement dit, elle n'est plus considérée comme la loi et le guide auxquels tous doivent se conformer ${ }^{30}$. Ici, Calvin commence à considérer la discipline en relation avec son rôle de prévention et de contrôle de la transgression des normes ecclésiales et morales ; c'est-à-dire qu'il commence à la décrire comme un pouvoir judiciaire disciplinaire (pénal). Puisque l'Église a besoin de la discipline plus que les 
autres communautés, étant donné qu'on attend d'elle un ordre plus grand que celui que l'on attend des autres associations humaines ${ }^{31}$, elle doit donc se purifier des délits commis par ses fidèles et ses ministres, que ces délits touchent aux Deux Tables ou aux lois cérémoniales et disciplinaires.

La discipline des ministres ayant déjà été définie par Calvin dans les Ordonnances ecclésiastiques de 1541, il se limite maintenant à nous la rappeler. Le pouvoir disciplinaire dérive du pouvoir des clés ${ }^{32}$, indispensable pour écarter de la communauté ceux qui vivent de façon immorale et impie et éviter la contamination du reste des fidèles. L'objectif de la loi vise donc tout à la fois l'édification et la punition ${ }^{33}$ : le châtiment va disposer le pécheur à renoncer à ses vices et le rendre à la communauté, convenablement réhabilitée ; pour les témoins du châtiment, celui-ci sera une bonne leçon qui va leur permettre de ne pas commettre la même erreur.

Mais, pour que l'Église puisse faire ce travail disciplinaire, elle doit se servir des moyens nécessaires, qui incluent l'excommunication, le plus puissant de tous ${ }^{34}$. L'ordre que poursuit la discipline ecclésiale n'est pas celui de la communauté civile ou celui de la vie domestique. Son objectif principal est de créer les conditions nécessaires pour que la Parole de Dieu soit décemment prêchée, cest-à-dire que sa priorité est celle du maintien de l'orthodoxie doctrinale et morale. De toute façon, la nécessité de la discipline va de pair avec la nécessité de l'autorité, tant pour organiser la communauté que pour la préserver, en éliminant les conduites qui la mettent en danger. Cependant, comme le dit à juste titre Höpfl, la question ici n'est pas seulement la nécessité de la discipline, mais l'absolue nécessité qu’elle revienne entre les mains de l'Église : on n’a pas seulement besoin d'un censeur, on a besoin d'un censeur clérical ${ }^{35}$. Et le pouvoir de juger doit revenir non pas à un juge, mais à une institution : le Consistoire.

Ainsi, la théorie devient réalité : on voit les idées de Calvin sur l'organisation ecclésiale et le crime dépasser le cadre théorique de l'Institution pour s'enraciner dans la cité de Genève sous la forme des Ordonnances ecclésiastiques. L'importance de cette organisation disciplinaire peut se mesurer en analysant l'activité de l'institution créée pour la mettre en pratique : le Consistoire ${ }^{36}$, fondé en décembre 1541, suite à la publication des Ordonnances.

Le Consistoire était composé de laïcs et de ministres, tous salariés de l'État genevois. Il s'agissait d'une institution conçue à la façon d'un sénat ecclésial, dont l'objectif était d'instruire le peuple dans les bonnes mœurs. Même s'il était un organisme ecclésial, ses séances, au début, étaient présidées par un 
syndic représentant les autorités civiles, incohérence que le Petit Conseil ou Conseil d'État fait disparaître en 1560, moment où l'on décide que le pouvoir du président du Consistoire doit être uniquement spiritue ${ }^{37}$.

Höpfl a raison, à notre avis, lorsque il souligne que l'argumentation juridico-disciplinaire calvinienne est soutenue par une sorte de syllogisme pratique aristotélicien ( $E N$ VI, 11, 1144a31) : la prémisse majeure nous indique que dans la circonstance de type $\mathrm{A}$, le chrétien doit faire $\mathrm{X}$; si la prémisse mineure indique qu'on est dans la circonstance de type $\mathrm{A}$, la conclusion renvoie à l'obligation de faire $\mathrm{X}$; dans le cas contraire, on recevra le châtiment correspondant ${ }^{38}$. Tout cela indique qu'il est nécessaire que l'acte - prémisse mineure - puisse être considéré comme un délit pour être jugé, c'est-à-dire qu'il soit inclus parmi les circonstances prévues par la prémisse majeure. C'est seulement de cette façon qu'il sera possible d'attribuer au sujet la responsabilité de son action. Par conséquent, le talon d'Achille de l'application judiciaire du syllogisme résidait dans l'interprétation de la prémisse mineure. Il est vrai cependant que le champ du délit moral va s'agrandir énormément puisque Calvin va se servir non seulement des Ordonnances ecclésiastiques, mais aussi de l'Écriture en tant que source de prémisses majeures - c'est-à-dire des actions réglées ou interdites, explicitement ou implicitement, par le Législateur. Je renvoie à l'herméneutique de la synecdoque qui sert à Calvin de guide d'interprétation.

Et, en effet, cela s'est passé ainsi. Au début, la marche du Consistoire était inspirée par le tribunal matrimonial de Berne, de façon telle que les problèmes traitaient, principalement, de la sexualité et de la validité des promesses matrimoniales $^{39}$. De cette façon, les fautes morales provoquant le plus de poursuites, et le plus souvent jugées, étaient en relation avec l'adultère. Il ne faut pas oublier que le sexe en dehors du mariage mettait en danger l'institution même puisque, en dépit du fait qu'il n'était plus un sacrement, le mariage conservait, pourtant, un caractère ecclésial ${ }^{40}$. La famille devait aussi être protégée et, avec elle, ses fruits : les enfants.

Lactivité du Consistoire genevois pendant ses premiers 24 mois est surprenante : ce ne sont pas moins de 850 personnes qu'il convoque au sein d'une population adulte de moins de 13000 individus ${ }^{41}$. Et, petit à petit, le rôle du Consistoire va sétendre jusquà la surveillance de la conduite du citoyen en général. Il poursuit alors les ivrognes, les blasphémateurs, les usuriers et les mendiants ; il condamne les jeux, les danses, et les vêtements qu'il juge indécents ou inadaptés. On compte ainsi plus de 700 excommunications en 1560 - moment 
de l'apogée du calvinisme à Genève -, dont 160 prononcées pour adultère, 102 pour ivrognerie, 66 pour blasphème, 35 pour pratique du jeu, 33 pour pratique de la danse et 27 pour avoir joué le rôle d'usuriers ${ }^{42}$. Mais les procès consistoriaux ne nous informent pas seulement sur la culture et les mœurs genevoises; ils nous offrent aussi une carte minutieuse de la disposition religieuse du peuple, puisque le Consistoire était également chargé de réprimer les pratiques et croyances de la foi antique (catholique), menant à cette fin de véritables interrogatoires sur les prières, la fréquentation du culte, la compréhension des doctrines réformées, etc. De même, il s'occupait des disputes entre familiers ou voisins, non pas seulement dans le but de préserver l'ordre social mais aussi pour éviter la profanation de la communion, ce dernier point étant fondamental pour une religion qui avait renoncé à la confession auriculaire et à la pénitence.

Endoctrinement, traque des délits moraux, contrôle des conduites liées au mariage, au baptême et à la sainte Cène... Le Consistoire était, selon Robert Kingdon, un corps quasi-judiciaire (quasi-judicial body) dont les membres jouaient le rôle de juges ${ }^{43}$. Malgré tout, ses pouvoirs étaient limités puisque, dans les cas graves qui méritaient lemprisonnement, le bannissement ou l'exécution, sa fonction consistait uniquement à entendre les témoins et à préparer l'enquête dont il devait remettre les résultats au gouvernement civil, un Tribunal semblable au Grand Jury anglo-saxon ${ }^{44}$. De fait, les cas d'hérésie, tels que celui de Servet, étaient considérés comme des affaires criminelles dont seulement le pouvoir civil pouvait se charger. Cependant, le Consistoire pouvait administrer d'autres châtiments, tels que l'admonestation ou, comme nous l'avons déjà dit, l'excommunication.

Ce qui est intéressant, c'est qu'on peut mettre en parallèle les fautes passibles d'excommunication et les délits condamnés dans les Deux Tables de la Loi. En effet, comme nous le rappelle William Monter, malgré la variété des conduites pourchassées sous l'étiquette de scandale - et parmi lesquelles on trouve les délits doctrinaux -, le contrôle de l'orthodoxie ne fut le souci principal du Consistoire que pendant ses premières quinze années, tandis que dans les derniers temps de la vie de Calvin, son intérêt se concentra principalement sur le contrôle moral ${ }^{45}$. Le mensonge, les délits sexuels, le respect de l'autorité (parents, mari, pouvoirs ecclésiaux), l'harmonie familiale et les bonnes relations avec le prochain... autant de devoirs explicités dans la Deuxième Table et que le Consistoire traduit en autant de délits. Cette attention quasi exclusive aux seuls délits de la Deuxième Table peut signifier que l'accomplissement des 
devoirs de la Première Table était un fait plus ou moins acquis, tout au moins dans les cités ${ }^{46}$ : respect de la doctrine et du culte, abandon de la superstition et du papisme, respect de l'Évangile, etc. Dès lors, le travail du Consistoire va principalement se résumer à adapter le comportement des citoyens genevois aux valeurs chrétiennes ; il va pourchasser l'opposition des fidèles aux préceptes de la Deuxième Table, laquelle doit, comme Calvin l'avait écrit dans l'Institution, servir de guide de conduite.

Le crime et l'excommunication vont aller de pair à Genève pendant les dernières années de la vie du réformateur. Jamais, selon Monter $^{47}$, pendant les quatre cents ans d'existence du Consistoire, le contrôle moral n'a été aussi fort que durant les dernières années de la vie de Calvin. Peut-on dire la même chose de la sphère civile?

\section{Le monopole de la violence}

[Å la police civile] n'appartient pas seulement à ce que les hommes mangent, boyvent et soyent sustentez en leur vie, combien qu'elle comprenne toutes ces choses quand elle fait qu'ils pussent vivre ensemble, toutesfois elle n'appartient point à ce seulement, mais à ce qu'idolâtrie, blasphémes contre le nom de Dieu et contre sa vérité, et autres scandales de la religion, ne soyent publiquement mis en avant et semez entre le peuple $[\ldots]^{48}$.

On vient de voir que la loi morale constituait la seule règle de justice, valable en tout temps et lieux, à laquelle Dieu veut que les chrétiens se soumettent. Le caractère didactique de la loi morale permet alors de la définir comme le fondement d'une éthique chrétienne considérée comme la seule règle de justice. Ce modèle de justice devait être concrétisé par des lois conformes à son esprit ; telle était la fonction du magistrat chrétien.

De fait, on peut dire que l'objectif de l'État calvinien était de soumettre le peuple à la loi divine que le magistrat connaît à travers le Décalogue ${ }^{49}$. Or, là où les juristes français soumettent le magistrat à la loi en raison de l'origine populaire de celle-ci, Calvin soumet le magistrat à la loi en vertu de son origine divine. La mission du magistrat sera dêtre l'instrument et les mains de Dieu sur terre, afin de créer un gouvernement providentiel dans l'histoire. Pour ce faire, 
le magistrat peut dicter des lois (pouvoir législatif), des édits (pouvoir exécutif) et rendre la justice (pouvoir judiciaire) ${ }^{50}$. Ainsi, le magistrat doit veiller à ce que le chrétien vive conformément à la loi divine, sans attenter à son prochain ${ }^{51}$.

Selon Witte, l'État exercera la prévention à travers le droit pénal ; il obtiendra la rétribution de la faute et procurera la réhabilitation du criminel ${ }^{52}$. Dans le même sens, Allegretti maintient que la fonction principale que Calvin attribue au pouvoir judiciaire est de réconcilier les gens en litige ${ }^{53}$. Ce qui est certain est que Calvin insistait sur un modèle de justice effective dans lequel le magistrat avait le pouvoir dépée (ius gladii) pour défendre ceux qui vivaient conformément à l'Évangile et châtier ceux qui le transgressaient ${ }^{54}$.

Or, le droit civil pénal nest pas seulement un droit mais un devoir des autorités dans leur fonction de lieutenants de Dieu. Juger est une mission quasi divine que les magistrats exercent à l'image de Dieu ; voilà pourquoi ils peuvent même se servir de la peine de mort. De cette façon, la source du pouvoir pénal civil est la justice divine dont sont investis les magistrats ${ }^{55}$. De plus, il ne faut pas oublier que, aux yeux de Calvin, le rôle du magistrat consiste aussi à agir au nom de la religion et de Dieu lui-même ${ }^{56}$. À ceux qui réduisent sa fonction aux devoirs envers le prochain évoqués dans la Deuxième Table, le réformateur rappelle que, pour les chrétiens, la source et le fondement de la justice est la religio : les devoirs religieux du magistrat précèdent et présupposent ses devoirs sociaux. Cela veut dire que sa mission principale est de veiller sur l'honneur de Dieu et sur la vraie religion et ne doit en aucun cas être réduite aux devoirs sociaux ${ }^{57}$.

Le mandat du magistrat consistait à établir la religio et la aequitas dans la cité, c'est-à-dire qu'il était chargé de concrétiser les vertus dont parlent les Tables du Décalogue. Ainsi, les magistrats étaient considérés comme les gardiens jaloux de l'Église, au pouvoir assez grand pour avoir la possibilité de décider d'une guerre ${ }^{58}$, c'est-à-dire de pourchasser non seulement les criminels internes mais aussi l'ennemi externe mettant en danger la religiosité des citoyens.

\section{Conclusion}

Autour de 1970, Michel Foucault prônait une orientation généalogique capable de montrer comment les pratiques discursives, qui génèrent la connaissance, s'articulaient avec des pratiques non discursives, génératrices, 
quant à elles, du pouvoir. Dès lors, le concept de discipline n'apparaît pas comme un élément étranger à la philosophie, puisque Foucault s'en sert pour élaborer ses thèses dans Surveiller et punir, où il illustre la discipline moderne à travers les pratiques des prisons, la formation militaire et l'instruction scolaire ${ }^{59}$. Notre étude montre que Foucault sous-estimait l'articulation pré-moderne de la discipline, du moins si on se réfère à la Genève du XVI siècle.

Le concept de discipline appartient à l'ancienne tradition théologique chrétienne, où il apparaît associé à la question du péché et à celui de la pénitence; se référant au privilège établi en Matthieu 18, on considérait l'absolution des pêchés comme la prérogative exclusive de l'Église. Ainsi que le souligne Michael Walzer, seule la discipline peut assurer l'organisation d'une communauté de saints suite à la corruption de notre nature ${ }^{60}$. Cependant, c'est à l'État que Walzer confiait l'administration de la discipline. Or, on sait que tous les efforts de Calvin tendirent à réclamer ce pouvoir pour l'Église.

Ainsi, tout en rompant avec elle, Calvin poursuit une tradition qu'on peut rattacher au $\mathrm{IV}^{\mathrm{e}}$ siècle, moment où naquit le système pénitentiel propre au catholicisme. Il est très important de noter qu'il ne s'agit pas uniquement d'une théologie sacramentelle mais aussi d'un ensemble de pratiques qui génèrent $d u$ pouvoir. On ne peut pas expliquer l'importance institutionnelle de l'Église dans la vie publique de l'Occident médiéval sans être attentif au contrôle social que pouvaient exercer les prêtres.

La discipline peut alors se décrire comme un système juridique pénal ecclésial sans contrainte physique, qui a pour finalité le contrôle du péché et le maintien de l'ordre dans la communauté des fidèles. Le pouvoir ecclésial de contrainte était uniquement d'ordre spirituel : l'église pouvait réprimander et excommunier, mais elle n'avait pas le pouvoir de contrainte matériel ou physique en appliquant des peines telles que la torture, le bannissement, la prison ou la mort ${ }^{61}$; ces châtiments ne pouvaient être imposés que par le pouvoir civil.

Certes, le péché n'est pas la source de l'État (bien qu'il soit l'occasion de sa fondation), mais il est la cause du pouvoir de contrainte liée au droit pénal, ecclésial aussi bien que civil. Ces pouvoirs seront donc chargés de refréner les effets du péché. Donc, les visées du droit pénal civil et du droit pénal ecclésiastique sont les mêmes, mais ce n'est pas le cas de leurs moyens ; or, les peines sont en relation avec les moyens. Par conséquent, l'État autant que l'Église sont respectivement dépositaires du ius gladii, un droit pénal civil et disciplinaire. Tous deux sont juridiques, mais l'un d’entre eux seulement peut se réclamer de 
la peine corporelle, l'autre ne pouvant se réclamer que d'un châtiment spirituel; telle est la différence entre droit et discipline $e^{62}$.

Le pouvoir de contrainte appartient uniquement à l'État, et ni l'individu ni l'Église ne peuvent prétendre à se l'approprier. À la suite d'Ockam et de Marsile de Padoue ${ }^{63}$, Calvin est aussi un précurseur des thèses wébériennes selon lesquelles l'État avait le monopole de la violence légitime ${ }^{64}$.

\section{Notes}

* Cette publication a été rendue possible grâce au Programme d' aide financière à la recherche Los primeros pasos del laicismo : Spinoza y Bayle (FFI2010-15578). Larticle est dédié à $\mathrm{M}$. Carbonnier car ses travaux sur le droit de punir chez Calvin sont à lorigine de ma propre recherche sur cet aspect de l’ouvre du réformateur français. Je suis reconnaissante du travail de révision du français fait par Marlène Jaouich, secrétaire à l'Institut d'Histoire de la Réformation (Genève). Je remercie aussi les suggestions du rapporteur, puisqu'elles ont permit d’améliorer l'article. Je remercie aussi le réviseur de langue français de Renaissance et Réforme qui a patiemment révisé la version finale de cette étude.

1. Il ne faut pas prendre le rejet protestant du Magistère ecclésial pour la défense d’une interprétation subjective de la Bible. L'examen protestant met fin à l'harmonie préétablie entre l'Écriture et le Magistère catholique mais n’implique pas la liberté de conscience moderne. Pour Calvin et Luther, c'est la Bible qui impose son interprétation au sujet; ce n'est pas le sujet qui lui donne son sens. Je remercie MariaCristina Pitassi et Marc Vial à l'Institut d'Histoire de la Réformation (Genève) de leurs orientations/recherches sur cet aspect. M.-C. Pitassi est en train de finir un livre sur cette importante question : La construction protestante d'un mythe de la modernité : le libre examen de l'Écriture (Paris : Honoré Champion : à paraître).

2. D. Forrester, «Martin Luther and John Calvin », in éd. L. Strauss et J. Cropsey, History of Political Philosophy (Chicago: University Chicago Press, 1963-1972-1987) p. 318-356, p. 322.

3. Du même avis, le théologien reformé B. Reymond : cf. Entre la grâce et la loi. Introduction au droit ecclésial protestant (Genève : Labor et Fides, 1992), p. 43.

4. Abréviations utilisées dans l'article :

Bible Toutes les abréviations suivent la vulgata en latin. 
CO Calvin, Joannes Calvini opera quae supersunt omnia, éd. G. Baum, E. Cunitz, E. Reuss, (Braunschweig : 1863-1900).

Com. Commentaire de Calvin sur le texte biblique indiqué dans la citation, avec son emplacement dans CO.

IRC Jean Calvin, Institution de la religion chrestienne, 5 vols., éd. critique de J.-D. Benoît sur l' édition définitive de 1560 (Paris : Vrin, 19571963).

PL J. P. Migne, Patrologie Latine, 221 vols., (Paris : J. P. Migne, 1844-1865).

RC R. Kingdon, dir., Registres du Consistoire de Genève au temps de Calvin, vols. 1-2 (Genève : Droz, 1996-2001).

RCP R.-M. Kingdon et J.-F Bergier, dirs., Registres de la Compagnie des Pasteurs de Genève au temps de Calvin [Genève : Droz, vol. 1 : 15461553, (1964) ; vol. 2 : 1553-1564 (1962)].

Serm. Sermon de Calvin sur le texte biblique indiqué dans la citation, avec son emplacement dans CO.

WA Luther, Weimarer Ausgabe. Nous nous sommes servis de l'édition O. Clemen, Luthers Werke. Studienausgabe, 4 vols. (Berlin : De Gruyter, 1983).

5. IRC II, 7, 1 .

6. Thèse qui va incorporer en 1559, dans l'article 23, la Confession de foi des Églises reformés de France, nommée Confession de la Rochelle, qui sétait inspirée de Calvin.

7. IRC II, 8,1 .

8. Cette division tripartite ne vient pas de Luther mais de Melanchton : cf. Loci Comunes rerum theologicarum seu hypotheses theologicae, 1546 (ed. or. 1521), p. 190 et ss.

9. Calvin utilise plutôt usus theologicus que elenchticus.

10. IRC II, 7, 7 et Com. Rom. III, 19 (CO 49, 55-56). Ainsi, Com. 1 Cor. 3, 7 (CO 50, 42).

11. IRC II, 7, 10 .

12. IRC II, 7, 13, souligné par nous. Presque avec les mêmes mots chez IV Serm. Deut., 32-35 (OC 26, 209).

13. IRC II, 8,6 .

14. IRC II, 8,11 .

15. Je me suis occupé de la discussion de l'éthique chez Calvin dans un autre article : M. García-Alonso " Biblical Law as the source of morality in Calvin », History of Political Thought 32 (2011), p. 1-19. 
16. De lib. arb. I, 15, 32 .

17. STh I-II q100 a 9.

18. IRC II, 8, 6 .

19. Calvin dénonçait lui-même l’identification de la loi mosaïque à la loi de l'État (IRC IV 20, 14).

20. IRC IV, 10, 30. C'est nous qui soulignons.

21. «Ie parle seulement de la puissance spirituelle, laquelle est propre à l'Église. Or icelle consiste en trois membres : assavoir en la doctrine, ou en la iurisdiction, ou en la faculté d'ordonner loix et statuts » (IRC IV, 8, 1). Wendel avait reconnu qu'il s'agissait de lois dérivées de la même église. Cf. F. Wendel, Calvin (Paris : Presses Universitaires de France, 1950), p. 233. Dans le même sens, J.-C. Groshens, Les institutions et le régime juridique des cultes protestants (Paris : R. Pichon et $\mathrm{R}$. Durand-Auzias, 1957), p. 34.

22. IRC IV, 10, 1. Sur ces aspects, cf. P. Le Gal, Le droit canonique dans la pensée dialectique de Jean Calvin (Fribourg : Éditions Universitaires Fribourg, 1984), surtout p. 142 et ss.

23. P. Prodi, Una storia della giustizia. Dal pluralismo dei fori al moderno dualismo tra coscienza e diritto, (Bologna : Il Mulino, 2000). Je me suis servi de l'édition espagnole : Una historia de la justicia. De la pluralidad de fueros al dualismo moderno entre conciencia y derecho (Buenos Aires-Madrid: Katz, 2008), p. 215.

24. «À l'époque carolingienne coexistent ainsi deux modalités de pénitence : les péchés secrets sont remis secrètement par un prêtre au moyen d'une pénitence tarifée ; les péchés publics sont effacés par la lourde procédure de pénitence publique. Ce système est profondément remis en cause au XII ${ }^{\mathrm{e}}$ siècle, notamment par la pratique de la confession auriculaire, rendue obligatoire par le $\mathrm{IV}^{\mathrm{e}}$ Concile de Latran en 1215 » (cf. R. Eckert, La peine en droit canonique du Decret de Gratien (vers 1140) au IVe Concile de Latran (1215), Mémoire de Maîtrise dirigé par L. Mayali et présenté à l'École Pratique des Hautes Études (inédite), 2007, p. 37. Je remercie l'auteur qui m’a fait, avec générosité, parvenir son magnifique travail.

25. Cf. R. Eckert, p. 12.

26. Pour un survol de l'histoire de l'adage, voir Eckert, La peine en droit canonique du Decret de Gratien..., p. 72 et ss.

27. Prodi, Una historia de la justicia..., p. 228.

28. "Non enim consisteret peccatum, si interdictio non fuisset. " (Pierre Lombard, Sententiarum Libri Quatuor, PL v. 192, cc. 519-964; la citation se trouve dans la c. 734) 
29. À l'exception de quelques paragraphes qui appartiennent à l'édition de 1541 et de quelques lignes qui correspondent à l'édition de 1560, le chapitre de 50 pages fut écrit dans sa totalité en 1545. Dans l'édition de 1560, c'est le chapitre XII : De la discipline de l'Eglise dont le principal usage est aux censures et en l'excommunication (IRC, éd. J. D. Benoît, p. 238-264).

30. IRC IV, 12, 1. Parallèlement, les lois civiles sont décrites en tant que nerfs de la communauté politique (IRC IV 20,14). L'expression a servi à montrer l'importance de la discipline calvinienne : cf., R. A. Mentzer, «Disciplina nervus ecclesiae : The Calvinist Reform of Nîmes », Sixteenth Century Journal 18 (1987), p. 89-116.

31. IRC IV 12, 1.

32. En parlant de Mt 18 : IRC IV 12, 1.

33. Certes, les mêmes fonctions étaient exposées par saint Augustin ; elles furent acceptées par Gratien et seront reprises par les canonistes (cf. Eckert, p. 102).

34. IRC IV 11, 1. Pourtant, dans les Ordonnances ecclésiastiques de 1541, le magistrat avait ajouté au projet originel de Calvin un article contenant la réduction de la juridiction ecclésiastique à la prédication de l'Évangile (Ordonnances ecclésiastiques, RCP I, p. 13). Le projet d'origine est dans CO 10, 15-30. Nous nous sommes servi de l'édition incluse dans RCP I, p. 1-13 puisque cette édition promulguée par les magistrats inclut avec les ajouts du pouvoir civil au texte du réformateur.

35. Cf. H. Höpfl, The Christian polity of John Calvin (Cambridge: Cambridge University Press, 1985), p. 115.

36. Sur le Consistoire genevois, cf. R. Kingdon, "The Control of Morals in Calvin's Geneva », in éd. L. P. Buck \& J. W. Zophy, The Social History of the Reformation (Columbus : Ohio State University Press, 1972), p. 3-16, en particulier p. 13, T. Lambert, Preaching, Praying and Policing the Reform in Sixteenth Century Geneva (Ann Arbor : Michigan, University Microfilms International, 2000), p. 237-78; W. Monter, « The Consistory of Geneva, 1559-1569 », Bibliothèque d'Humanisme et Renaissance 38 (1976), p. 467-484. Le rapporteur a attiré mon attention sur l'importance de discuter plus largement de la pratique du Consistoire genevois et de sa fonction juridique sociale. Je m'en suis occupé dans un autre article : $\mathrm{M}$. García-Alonso, "Calvin and the Ecclesiastical Power of Jurisdiction », Reformation and Renaissance Review 10.2 (2008), p. 137-155.

37. Un geste accompagnait cette décision : pour faire partie du Consistoire, le président renonçait au bâton de syndic, symbole de la juridiction civile (OC X col. 121122).

38. Höpfl, p. 177-78. 
39. Le Consistoire de Berne (1528), exclusivement seigneurial (Consistoire seigneurial), était une institution civile qui détenait pourtant le pouvoir d'excommunication : cf. T. Lambert et I. M. Watt, «Introduction », in Registres du Consistoire de Genève au temps de Calvin, vol. I (1542-1544), p. 19.

40. Calvin était très sensible aux problèmes du mariage ; pour preuve les Ordonnances matrimoniales, qu'il rédige en 1545, mais qu'on ne publie pas jusqu'à la nouvelle édition des Ordonnances ecclesiastiques de 1561 (CO 10, 92-124). Sur le mariage, cf. R. Kingdon, Adultery and Divorce in Calvin's Geneva (Cambridge, Mass. : Harvard University Press, 1994).

41. Cf. T. Lambert et I. M. Watt, «Introduction », in Registres du Consistoire de Genève au temps de Calvin, vol. I (1542-1544).

42. Cf. W. Monter, "La Réforme au quotidien ", in dir. P. Chaunu, L'Aventure de la Réforme (Paris : Hermé, 1986), p. 245 et ss.

43. Cf. R. Kingdon, Adultery..., p. 17.

44. Cf. R. Kingdon, Adultery..., , p. 16.

45. Cf. W. Monter, « The Consistory... », p. 475.

46. Cf. R. Mentzer, « Le Consistoire et la pacification du monde rural », Bulletin de la Société de l'Histoire du Protestantisme Français 135 (1989), p. 373-389.

47. Cf. W. Monter, "The Consistory... », p. 484. Voir aussi R. Kingdon, qui va dans le même sens : "The Control of Morals... », p. 12.

48. IRC IV 20, 3. Le texte poursuit de cette façon : "Car ie ne permets icy aux hommes de forger loix à leur plaisir touchant la religion et la maniere d'honnorer Dieu, non plus que ie faisoye par cy devant : combien que i'approuve une ordonnance civile, laquelle prend garde que la vraye religion qui est contenue en la Loy de Dieu, ne soit publiquement violée et pollué par une licence impunie » (ibid.).

49. Cf. M.E. Chenevière, La pensée politique de Calvin (Genève, Paris : Éditions Je Sers, 1937), p. 171.

50. «En quelle hardiesse prononceront-ils sentence iniuste de leur bouche, laquelle ils cognoistront estre destinée pour estre organe de la vérité de Dieu ? En quelle conscience signeront-ils quelque mauvaise ordonnance de leur main, laquelle ils sauront estre ordonée pour escrire les arrests de Dieu ?» (IRC IV 20, 6).

51. I Serm. Deut. 20, 1-4 (CO 27, 588-602).

52. Cf. J. Witte "The Three Uses of the Law: a Protestant Source of the Purposes of Criminal Punishment ? », Journal of Law and Religion 10 (1994), p. 433-465, en particulier p. 452-457 ; J. Witte, « Moderate religious liberty in the Theology of John Calvin », Calvin Theological Journal 31 (1996), p. 359-403. 
53. Cf. J. Allegretti, " "In all this love will be the best guide". John Calvin on the Christian's Resort to the Secular Legal System », Journal of Law and Religion 9 (1991), p. $1-16$.

54. Com. 1 Tim. 2, 2 (CO 52, 266-68), Com. Rom. 13, 4 (CO 49, 251). Ainsi, IRC IV, 20,4 .

55. «[L]eur vocation n'est pas chose profane n'estrange d'un serviteur de Dieu, mais une charge tressaincte, veu qu'ils font mesme et exécutent l'office de Dieu. » (IRC IV, 20, 6). Sur le droit pénal calvinien, cf. M. Carbonnier, « Le Droit de punir et le sens de la peine chez Calvin ", Revue d'Histoire de Philosophie Religieuses 2 (1974), p. 187-201, en particulier p. 190, et Le droit de punir chez Calvin (Mémoire de Maîtrise, Universté de Paris, 1970). Je remercie M. Carbonnier de m’avoir offert ces travaux, qui sont à l'origine de ce travail. Cf., aussi, M. E. Chenevière, La pensée politique de Calvin, p. 276 et ss.

56. Com. 1 Tim. 2, 2 (CO 52, 266-268).

57. IRC IV 20, 9.

58. IRC IV, 20, 5 ; IRC IV, 11, 16 ; IRC IV, 20, 11.

59. M. Foucault, Surveiller et punir (Paris : Gallimard, 1993).

60. Cf. M. Walzer, La révolution des saints, trad. de V. Giroud, (Paris : Belin, 1987), ch. 2 .

61. À la différence de la tradition catholique, qui n’hésite pas à défendre la peine maximale, comme nous le rappelle Eckert : "Ainsi, pour les canonistes de la fin du XII ${ }^{\mathrm{e}}$ siècle, la peine revêt une fonction doublement préventive. Elle doit, par son exemplarité, inspirer la crainte aux délinquants potentiels. Elle prévient également la récidive du criminel. Le juge ne doit ainsi pas hésiter à prononcer la peine de mort, laquelle, pour les canonistes, ne revêt aucune dimension d'expiation mais offre simplement la meilleure garantie possible contre la récidive » (cf. Eckert, p. 119).

62. Dans le droit romain existait la potestas et l'auctoritas, toutes deux juridiques, mais pas de la même façon. En effet, dans la République consulaire, les Consuls fournissaient l'élément légal en exerçant le pouvoir exécutif et militaire (potestas) tandis que le Sénat fournissait le conseil aristocratique et les conseils politiques (consilium) puisqu'il avait l'auctoritas; le peuple choisissait les magistrats et approuvait leurs lois, car il possédait la liberté (libertas).

63. Cf. Breviloquium de principatu tyrannico (1339-1340) et Defensor Pacis (1324). 
64. Max Weber, The Profession and Vocation of Politics in Weber: Political Writings (Cambridge: Cambridge University Press, Cambridge Text in the History of Political Thought, 1994), p. 310 